\title{
Optimal Control of an Epileptic Neural Population Model ${ }^{\star}$
}

\author{
Justin Ruths* Peter Neal Taylor ${ }^{* *}$ Justin Dauwels ${ }^{* * *}$ \\ * Singapore University of Technology and Design \\ (e-mail: justinruths@sutd.edu.sg) \\ ** Nanyang Technological University, Singapore \\ (e-mail:ptaylor@ntu.edu.sg) \\ *** Nanyang Technological University, Singapore \\ (e-mail:jdauwels@ntu.edu.sg)
}

Neural control; Optimal control; Computational methods.

\begin{abstract}
Neural population models describe the macroscopic neural activity that can be clinically recorded by an electroencephalogram (EEG). Such models are relevant for the investigation of many pathological neurological phenomena including epilepsy and Parkinsons disease because the models operate on the same scale as the recorded data. Although several models exist in the neuroscience literature, none have leveraged the systematic approach of optimal control theory to design stimuli to treat such neurological conditions. In addition, these models have not yet reached the control community, which has instead largely focused on single neuron models. Here we present the model and a formulation of the seizure abatement goal expressed as an optimal control problem. We show several results including a realistic, noisedriven simulation where the control is applied as needed in a moving window.
\end{abstract}

\section{INTRODUCTION}

Epileptic seizures are transient neurological events which can occur up to hundreds of times per day in some patients (Blumenfeld and Taylor [2003]). Symptomatic manifestations of these events can include a loss of consciousness, tonic-clonic convulsions and myoclonic jerks, among others which can severely impact patient health and quality of life. These seizure events often have distinctive electrographic correlates detectable on an electroencephalogram (EEG). One commonly observed electrographic seizure waveform morphology is the spike wave discharge (SWD), which is characterized by a fast spike followed by a slow wave. SWDs are periodic oscillations with a frequency typically slower than that of normal awake EEG. They are frequently associated with absence seizures, myoclonic seizures and complex partial seizures (Taylor and Baier [2011]).

Although the first line of treatment for patients with epilepsy is typically medication, in over $30 \%$ of cases medication alone is insufficient (Keränen et al. [1988]). Brain stimulation via electrodes has been suggested as an alternative therapeutic treatment for epilepsy (Liang et al. [2010, 2012], Saillet et al. [2012]) and has had success in treating other neurological disorders including the motor symptoms of Parkinson's disease (Limousin et al. [1998], Feng et al. [2007]).

Mathematical models of neural field dynamics enable the analysis and prediction of system behavior. The recent literature in epilepsy has developed several mathematical

\footnotetext{
* This work was supported in part by the SUTD-MIT International Design Centre [Grant IDG31300103] (Ruths) and the Singapore Ministry of Education [Tier 1 Grant M4010982.040 RG37/11] (Dauwels).
}

models of SWD on the macroscopic scale at which EEG recordings are made (Breakspear et al. [2006], Marten et al. [2009], Goodfellow et al. [2011], Taylor and Baier [2011], Wang et al. [2012], Taylor et al. [2013c,a]). Progress on deriving stimuli for seizure abatement for such models, however, has not kept in step with this advancement in model refinement. In particular, more advanced methodology from optimal control theory has largely been absent.

Some previous studies have investigated the impact of stimulation and control in models with pathological activity. For example, Tass [2003] demonstrated a technique to disrupt synchrony in coupled phase oscillators, modeling the synchronous phenomena underlying Parkinson's disease. Kramer et al. [2006] used PID-type feedback controllers to effectively shift a model parameter to drive the system away from regions of parameter-space corresponding to epileptiform oscillations. Suffczynski et al. [2004] demonstrated in a bistable model that single pulse perturbations can abate abnormal epileptiform activity. Schiff [2010] suggested a model-based controller in Parkinson's disease with further potential expansions to epilepsy.

Although progress has been made, there is a distinct opportunity to apply the systematic approach provided by systems and control theory - in particular, computational optimal control theory - to neural population models. A number of important factors influence why open-loop optimized controls are a preferred solution in neuroscience. In feedback controllers, the stimulus is always "on", whereas optimal solutions can be triggered and then turned off afterwards - in the long run this leads to a less invasive solution. Similarly, optimal control allows us to generate minimal stimuli (e.g., minimum energy) that again are least invasive. A common criticism of open-loop control 
is the lack of robustness in optimal solutions, however, the recent advancements in optimal ensemble control theory provide mechanisms to overcome this deficiency in many scenarios ( $\mathrm{Li}$ et al. [2011]).

Within the control community, several groups, including Moehlis et al. [2006] and $\mathrm{Li}$ et al. [2013], have applied these systematic methods, however, have focused mainly on phase-models, which are highly reduced models of individual neurons. Since most patient data is collected by EEG, which operates at the macroscopic scale, in this work we use a more clinically relevant neural population model.

In the sections that follow, we present the macroscopic neural population model, formulate seizure abatement as an optimal control problem, and outline the computational method employed to solve the optimal control problem. We present several results which provide insight into the control of the model and also a sliding window controller which emulates the robustness of the solution under a realistic situation.

\section{METHODS}

\subsection{Model}

Experimental evidence suggests important roles for both the cortex and thalamus in the genesis and maintenance of epileptic SWD oscillations (Destexhe [1998], Pinault and O'Brien [2005]). We therefore incorporate knowledge of these anatomical structures into our model using neural field equations based on the Amari framework (Amari [1977], Taylor et al. [2013b,a]). The cortical subsystem is composed of excitatory pyramidal (PY) and inhibitory interneuron (IN) populations. The thalamic subsystem includes variables representing populations of thalmocortical relay cells (TC) and neurons located in the reticular nucleus (RE). All populations are interconnected in agreement with experimentally known connections (Pinault and O'Brien [2005]) using the connectivity parameters $C_{i}, i=1, \ldots, 9$. The resulting excitation model for (PY,IN,TC,RE) neurons, denoted $\left(x_{1}, x_{2}, x_{3}, x_{4}\right)$, respectively, is given as follows,

$$
\begin{aligned}
& \dot{x_{1}}(t)=\tau_{1}\left(h_{1}-x_{1}+C_{1} f\left[x_{1}\right]-C_{3} f\left[x_{2}\right]+C_{9} f\left[x_{3}\right]\right)+u(t) \\
& \dot{x_{2}}(t)=\tau_{2}\left(h_{2}-x_{2}+C_{2} f\left[x_{1}\right]\right)+u(t) \\
& \dot{x_{3}}(t)=\tau_{3}\left(h_{3}-x_{3}-C_{6} f\left[x_{4}\right]+C_{7} f\left[x_{1}\right]\right) \\
& \dot{x_{4}}(t)=\tau_{4}\left(h_{4}-x_{4}-C_{4} f\left[x_{4}\right]+C_{5} f\left[x_{3}\right]+C_{8} f\left[x_{1}\right]\right)
\end{aligned}
$$

where $h_{i}$ and $\tau_{i}, i=1,2,3,4$, are input parameters and timescale parameters, respectively; $u(t)$ is the applied stimulus; and $f[\alpha]$ is the sigmoid function,

$$
f[\alpha]=\left(1 /\left(1+\varepsilon^{-\alpha}\right)\right),
$$

where $\varepsilon$ determines the sigmoid steepness. This follows the connection schematic as shown in Fig. 1. Notice that the stimulus is applied only to the cortical variables PY and IN because this corresponds to a less invasive, more realistic scenario, since the thalamic structures are located deeper inside the brain.

We use parameters which place the model in an excitable state, namely $C=(1.8,4,1.4,0.2,10,1.5,3,3,1), h=$ $(-0.35,-3.4,-2,-5), \tau=(1,1.25,0.1,0.1)$, and $\varepsilon=$ 250000. In essence a threshold exists in the system which,

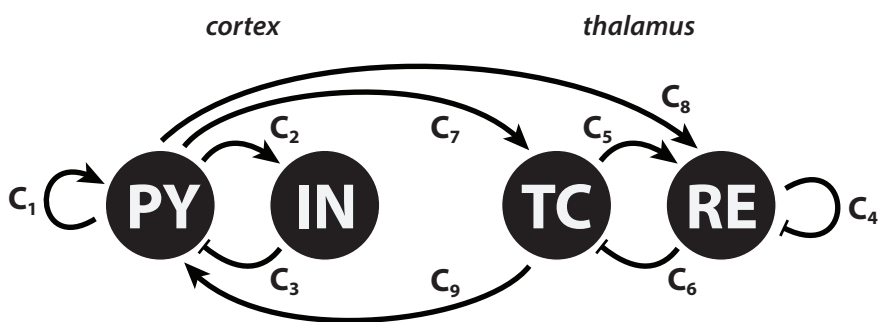

Fig. 1. Connectivity scheme of the model. Excitatory (inhibitory) connections indicated with arrows (buttons).

when a sufficiently strong supra-threshold stimulus is applied, can drive the dynamics to undergo transient SWD. We can, therefore, provoke a seizure in our model by applying a large enough stimulus. Model solutions are computed numerically using a fixed step Euler-Maruyama solver using MATLAB (Kloeden and Platen [1992]).

These nonlinear dynamics evidence a stable focus representing the non-seizure equilibrium state, $x_{r}=(0.1691$, $0.1645,-0.0913,0.0032)$ for the chosen parameter values. Seizure abatement in such a model can be understood as driving the system from the region corresponding to the transient SWD oscillations to a neighborhood around the stable focus. This is a state transfer problem we can solve by formulating it as an optimal control problem. In practice it is valuable to consider minimum energy stimuli, therefore, we create a cost function which derives minimum stimuli with endpoint constraints that enforce the state transfer. The optimal control problem seeks an input $u(t)$ which minimizes the cost functional,

$$
\int_{0}^{T} u^{2}(t) d t
$$

over the time window $t \in[0, T]$ subject to the dynamics given in (1) and the endpoint constraints $x(T)=x_{r}$ and $x(0)=x_{0}$. Solving such a highly nonlinear optimal control problem is rarely tractable, so we turn to computational methods to find the input $u(t)$.

\subsection{Pseudospectral Method}

The pseudospectral method is a direct collocation method to transform an optimal control problem into a nonlinear programming problem. The pseudospectral method has been successfully applied in a variety of applications including guidance for aircraft, quantum control, and neuroscience (Fahroo and Ross [2008], Li et al. [2011, 2013]). The method employs a relationship between orthogonal Legendre polynomials, which permit spectral accuracy (i.e., only a small number of terms are needed to approximate the function), and interpolating Lagrange polynomials, which enable the collocation nodes of the interpolation approximation to be used directly in the subsequent nonlinear optimization. The former facilitates efficient computation and the latter facilitates ease of implementation.

For the sake of space, we defer the reader to these resources for a comprehensive description of the method ( $\mathrm{Li}$ et al. [2011]). In summary, we consider an optimal control problem with cost in Bolza form, 


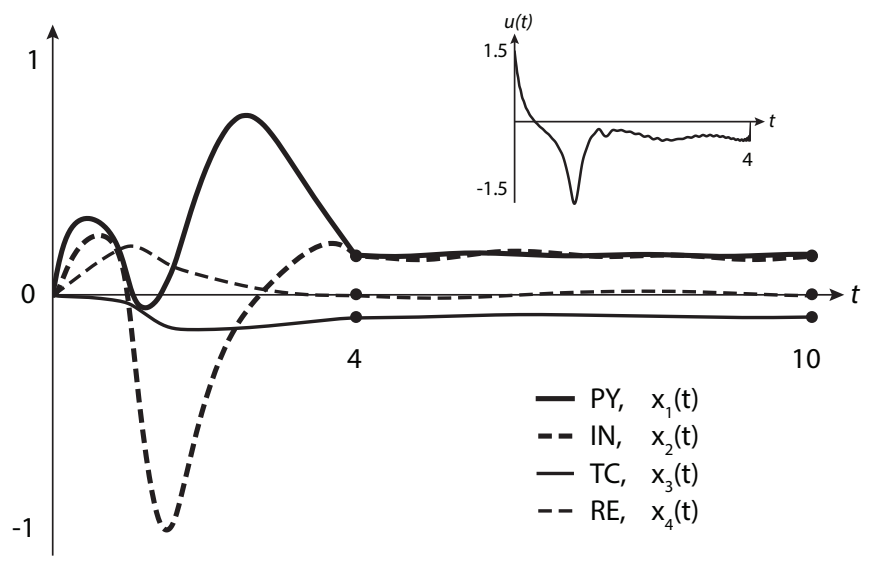

Fig. 2. Trajectories of the model driven by the optimal control (inset axis) in which terminal constraints, were imposed on all states, i.e., $e=x_{r}-x(T)$.

$$
\begin{array}{ll}
\min & \varphi(T, x(T))+\int_{0}^{T} \mathcal{L}(x(t), u(t)) d t, \\
\text { s.t. } & \dot{x}(t)=F(x(t), u(t)), \\
& e(x(0), x(T))=0, \\
& g(x(t), u(t)) \leq 0, \quad \forall t \in[0, T],
\end{array}
$$

where $x(t)$ and $u(t)$ is the state and control, respectively, at time $t ; \varphi$ and $\mathcal{L}$ are the terminal and running costs; $F$ is the dynamics; and $e$ and $g$ are endpoint and path constraints, respectively; is transformed to a nonlinear programming problem of the form,

$$
\begin{array}{ll}
\min & \varphi\left(T, \bar{x}_{N}\right)+\frac{T}{2} \sum_{i=0}^{N} \mathcal{L}\left(\bar{x}_{i}, \bar{u}_{i}\right) w_{i}, \\
\text { s.t. } & \sum_{k=0}^{N} D_{j k} \bar{x}_{k}=\frac{T}{2} F\left(x_{j}, u_{j}\right), \\
& e\left(\bar{x}_{0}, \bar{x}_{N}\right)=0, \quad \forall j \in\{0,1, \ldots, N\}, \\
& g\left(\bar{x}_{j}, \bar{u}_{j}\right) \leq 0, \quad \forall j \in
\end{array}
$$

where $\bar{x}_{j}=x\left(t_{j}\right)$ and $\bar{u}_{j}=u\left(t_{j}\right), t_{j}$ is the $j^{\text {th }}$ interpolation point; $w_{i}$ are weights characteristic of quadrature approximations of integrals; and $D$ is a constant coefficient matrix determined by the order of approximation/discretization $N$. Nonlinear programming problems of this form can be solved by any number of commercial and open source solvers. In this work we employ a commercial solution using AMPL and KNITRO (Fourer et al. [2002], Byrd et al. [2006]).

\section{RESULTS \& DISCUSSION}

In this work we present four successively more sophisticated demonstrations of stimuli solutions that eliminate the pathological spike wave discharge seizure behavior in the presented model.

\subsection{Optimal Control}

In this first demonstration, we investigate the state transfer problem in a "controlled environment." At time $t=0$, we start the system at $x_{0}=(0,0,0,0)$, which if left uncontrolled will directly enter seizure oscillations. Given these

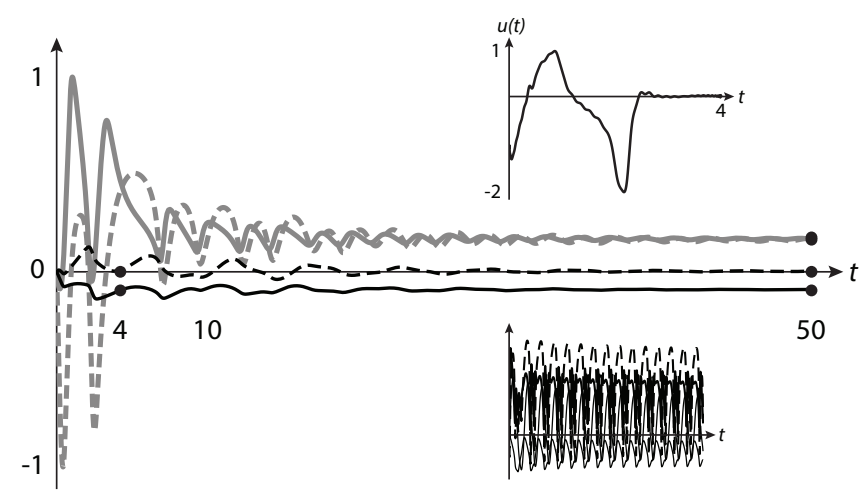

Fig. 3. Trajectories of the model driven by the optimal control (upper inset axis) in which terminal constraints were imposed on TC and RE states only, i.e., $e_{i}=x_{r_{i}}-x_{i}(T)$ for $i=3,4$. Trajectories of the model (lower inset axis) in which terminal constraints were imposed on PY and IN states only, i.e., $e_{i}=x_{r_{i}}-$ $x_{i}(T)$ for $i=1,2$.

initial conditions, we solve the minimum energy optimal control problem with terminal constraint $x(T)=x_{r}$ (i.e., $\left.e=x_{r}-x(T)\right)$. In these optimizations the final time is fixed at $T=4$. This value is chosen arbitrarily and can be adjusted. Oscillations present a challenge practically when discretizing optimal control problems and a fixed duration ensures that our level of discretization $(N=71)$ is sufficient to capture the motion accurately.

Figures 2 and 3 show that optimized stimuli $(u(t)$ plotted on the upper inset axes) are able to drive the system to the resting equilibrium $x_{r}$, indicated by $\bullet$ symbols in the figures. In Fig. 3, we impose a terminal constraint on only the TC, $x_{3}(T)$, and RE, $x_{4}(T)$, states, whereas in Fig. 2 terminal constraints are imposed on all states. Although the cortical and thalamic subsystems mutually influence each other, Fig. 3 illustrates the importance of the thalamic subsystem. This effect is likely due to the large timescale separation, which in our model can be considered as conductance delays between the respective brain structures. Notably, seizure abatement can be achieved by only by imposing an endpoint criteria on the thalamus variables. In contrast we find that imposing terminal constraints on the PY, $x_{1}(T)$, and IN, $x_{2}(T)$, variables alone does not achieve the same effect (see lower inset axes of Fig. 3).

\subsection{Optimal Control with Arbitrary Initial Conditions}

In practice, the control needs to be applied "online" rather than predictably at the outset (i.e., the seizure will begin at some point into the observation, $t>0$ ) and starting at a state different from the origin. The first step forward is to generalize the previous results for any arbitrary initial condition $x(0)=x_{0}$.

We observe that during a seizure, the system state trajectory follows relatively consistently path while it oscillates. We, therefore, modify the initial condition equality constraint to enforce a starting state selected arbitrarily from the trajectory of the seizure oscillation. For example, Figure 4 demonstrates driving the system from $x_{0}=(0.30$, 


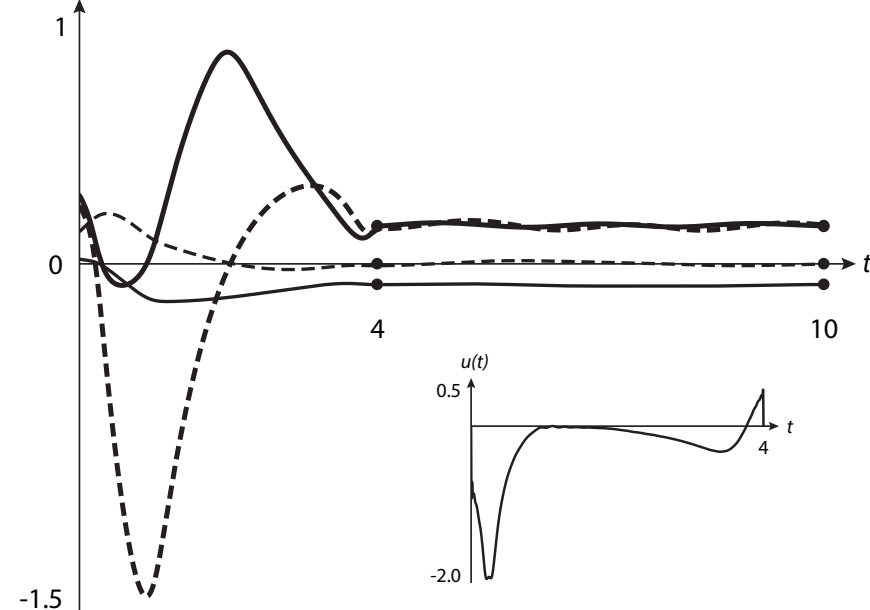

Fig. 4. Trajectories of the model driven by the optimal control (inset axis) starting from an arbitrary initial point contained in the seizure oscillations.

$0.25,0.02,0.14)$, which lies on the trajectory of the seizure oscillations, to $x_{r}$. Similarly as before, the stimulus is successful in aborting the seizure before it begins.

When we try to incorporate this stimulus into a moving window controller (as will be discussed in Section 3.4), in which the stimulus is triggered by a state-dependent criteria, we find that this solution no longer works properly. The controller triggers the stimulus when the state comes within a small neighborhood of the triggering point. In order for the stimulus to function consistently, it must be robust to variation in the initial state because any triggering mechanism will inherently introduce variation. In the context of this problem, this is enough to substantially affect the performance of the stimulus. With this motivation, we turn to ensemble control to develop robust open-loop stimuli.

\subsection{Ensemble Optimal Control}

Ensemble control is concerned with the study of systems which are structurally similar and driven by a common input, but differ by a parameter (Li et al. [2011]). Using this methodology we can formulate an optimal ensemble control problem, whose solution is robust to the variation in the parameter or parameters. Ensemble control has been employed to compensate for actual observed heterogeneity as well as uncertainty in the parameters. Because the initial condition of the system can be seen as a parameter of the dynamics, we can use this framework to develop stimuli robust to the starting state (Ruths and Li [2012]).

Similar to the case above, we select a portion of the trajectory traced out by the seizure oscillations, which we denote $\Omega$. We sample this section of the curve and create an optimal ensemble control problem which seeks to simultaneously drive $N_{\Omega}$ systems starting at various points along the trajectory to the stable equilibrium with a single common stimulus. The pseudospectral method provides a natural extension to implement and solve the ensemble optimal control problem (Li et al. [2011, 2013]).

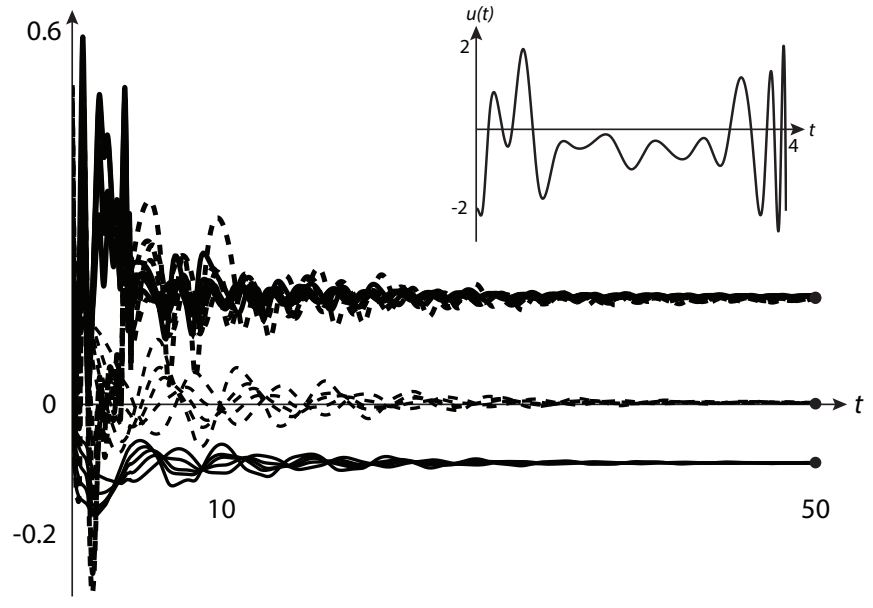

Fig. 5. Trajectories of 5 systems with different initial conditions $x_{0} \in \Omega$ driven by the common optimal ensemble control (inset axis).

Figure 5 displays the trajectories corresponding to five $\left(N_{\Omega}=5\right)$ different starting locations which are simultaneously driven to a neighborhood of $x_{r}$ by the common ensemble control, plotted on the inset axis. Therefore, this stimulus is able to compensate for the variation in starting state, which makes it significantly more capable of maintaining its efficacy when applied as an online controller.

\subsection{Moving Window Seizure Abatement}

We now develop a realistic simulation of seizure abatement using the model and the results described in the previous sections. In this case, the model is also driven, namely the TC $\left(x_{3}\right)$ dynamics, by an additional noise term, which represents both the measurement noise and the inherent background noise in the brain. Although the noisy input alone is sufficient to start a seizure, we add on top of this noise a small extra stimulus, or "kick", to reliably induce a seizure. This allows us to know that the seizure will occur within a given time horizon, which is convenient for practical reasons. Noise induced seizures are identical in overall shape and pattern, so inducing the seizure is not seen as artificial. More importantly, the kick allows us the opportunity to more systematically validate our ensemble control stimulus under a variety of onset parameters. Although the systems eventually reach the same seizure oscillation cycle, the transient behavior following a kick is more unique and related to the timing, the amplitude, and the duration of the kick. The ensemble control should be robust to any of these types of transient behavior.

In this scenario, we employ a moving window controller which monitors the system state in real-time. When a seizure is detected and the state approaches within a neighborhood of $\Omega$ (i.e., $\|x(t)-v\| \leq \epsilon$ for all $v \in \Omega$ ), the ensemble control is triggered and drives the state to the stable equilibrium, thereby stopping the seizure. Figure 6 compares the PY states of the uncontrolled and controlled system using the stimulus developed in Section 3.3 (up to now we have dealt with time in a dimensionless scale, now we represent this in physical time - i.e., in seconds). 

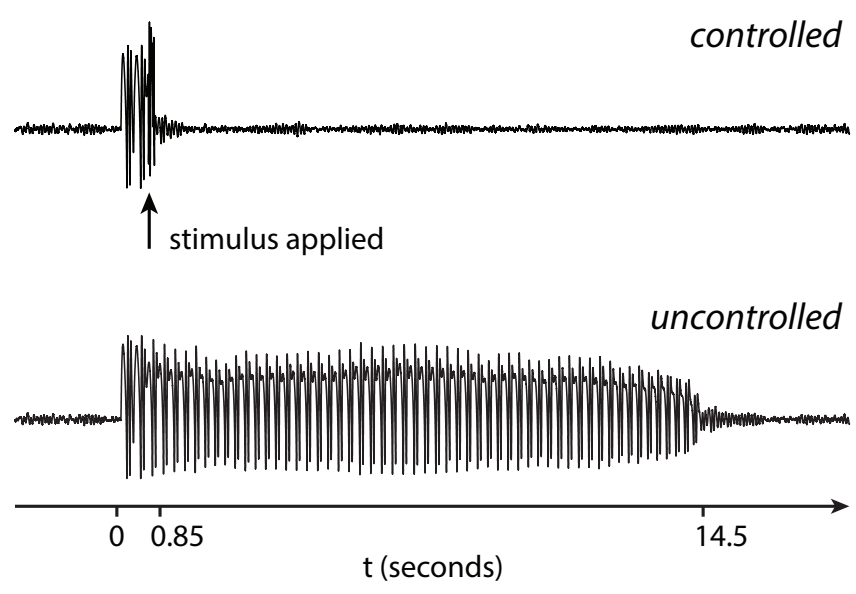

Fig. 6. The moving window controller detects and stops seizures as they occur. The PY variable, $x_{1}(t)$, is plotted for the controlled and uncontrolled cases.

Another limiting factor in practice is the ability to monitor all state variables. Determining the current state of the cortical variables, PY and IN, is significantly easier than observing the thalamic variables, TC and RE. Due to the fact that the thalamus is located in the middle of the brain, it is is extremely valuable (less invasive) if the seizure triggering mechanism only requires observations of the cortical variables. In addition, measurements can be taken using the stimulus probe, therefore, the PY and IN variables can already be monitored. The ensemble control is also able to address this issue because the ensemble control is able to compensate for the variation of TC and RE along the seizure oscillation cycle at a specific chosen PY and IN. Therefore, all results of this section used a triggering method based only on PY and IN (including Figs. 6 and 7).

We tested the ensemble stimulus over a comprehensive range of kick parameters, from $\times 1$ up to $\times 4$ the nominal kick amplitude and kick duration. We also tested the response to shifting the kick by up to 1 second in time, which is longer than a single period of oscillation, because different portions of the dynamics are more susceptible to the kick than others. Over these range of parameters, the controlled seizures lasted between 0.8 and 2.6 seconds. Using the same parameters (and notably the same noise vector), the uncontrolled seizures lasted between 4.1 and 25.0 seconds. Because the controller is triggered by the cortical variables alone, it is possible that the stimulus is applied when the cortical states are within $\Omega$ but the thalamic states are not. When this happens, often the stimulus is applied twice, however, we consistently observe (see Fig. 7) that the seizure is stopped in significantly less time than when the control is applied compared to when it is not.

\section{CONCLUSION}

We have introduced to the control community a neuron population level model for epilepsy characterized by spike wave discharge type oscillations. This model offers novel opportunities to model and study epilepsy behavior at the

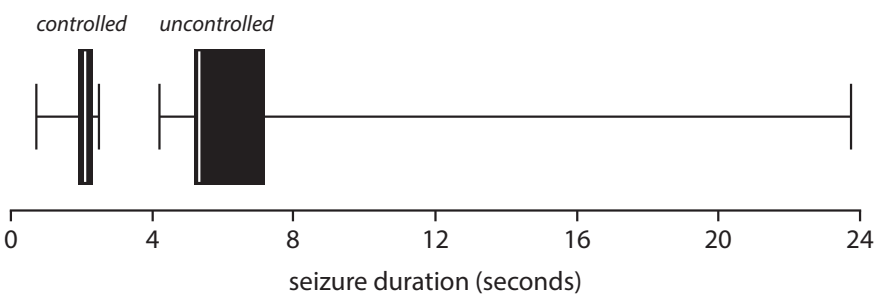

Fig. 7. Boxplots of the aggregate statistics for controlled and uncontrolled seizure durations over a wide range of kick parameters (delay: 0-1 second; amplitude and duration: $\times 1$ to $\times 4$ ).

scale of clinical data using the tools from dynamics and control theory. To the neuroscience community, this work provides a large step forward in developing methods which can be used to derive stimuli for neuroscience applications.

We demonstrate the flexibility and utility of the method by developing several types of stimuli, which offer intuition to the dynamics of the system and also introduce sequentially higher levels of clinical practicality. Our final example employs an ensemble control to flexibly drive the system from a region of seizure behavior to the stable resting point. This is done in a real-time moving window fashion, in which the controller monitors a subset of the system state and activates the stimulus when the system exhibits seizure behavior.

Both the model and the method admit straightforward generalizations to a spatially extended version in which each spatial cortical location is connected both to the thalamus, as is done in the present model, and also with other cortical locations. The weighting of these latter connections is given by a connectivity matrix, or network, representing the correlation in activity between the different cortical locations. We aim to expand the proof-of-concept reported in this article to address the spatially extended model.

\section{ACKNOWLEDGEMENTS}

The authors would like to thank Sydney Cash at Massachusetts General Hospital for helpful discussions regarding the model and Yujiang Wang and Nishant Sinha who provided support during the writing of the paper.

\section{REFERENCES}

S. Amari. Dynamics of pattern formation in lateralinhibition type neural fields. Biological Cybernetics, 27 (2):77-87, 1977. ISSN 0340-1200.

$\mathrm{H}$ Blumenfeld and J Taylor. Why do seizures cause loss of consciousness? The Neuroscientist, 9(5):301-310, 2003.

M. Breakspear, J.A. Roberts, J.R. Terry, S. Rodrigues, N. Mahant, and P.A. Robinson. A unifying explanation of primary generalized seizures through nonlinear brain modeling and bifurcation analysis. Cerebral Cortex, 16 (9):1296, 2006. ISSN 1047-3211.

R H Byrd, J Nocedal, and R A Waltz. Knitro: An Integrated Package for Nonlinear Optimization - Springer. Large-scale nonlinear optimization, 2006. 
A. Destexhe. Spike-and-wave oscillations based on the properties of GABAB receptors. The Journal of Neuroscience, 18(21):9099, 1998. ISSN 0270-6474.

F Fahroo and I M Ross. Pseudospectral methods for infinite-horizon nonlinear optimal control problems. Journal of Guidance Control and Dynamics, 31(4):927936, 2008.

X-J Feng, E Shea-Brown, B Greenwald, R Kosut, and $\mathrm{H}$ Rabitz. Optimal deep brain stimulation of the subthalamic nucleus-a computational study. J Comput Neurosci, 23(3):265-82, Dec 2007. doi: 10.1007/s10827007-0031-0.

R Fourer, D M Gay, and B W Kernighan. AMPL: A mathematical programming language. 2002.

M. Goodfellow, K. Schindler, and G. Baier. Intermittent spike-wave dynamics in a heterogeneous, spatially extended neural mass model. NeuroImage, 55(3):920-932, 2011. ISSN 1053-8119.

T Keränen, M Sillanpää, and P J Riekkinen. Distribution of seizure types in an epileptic population. Epilepsia, 29 (1):1-7, 1988.

Peter E Kloeden and Eckhard Platen. Numerical Solution of Stochastic Differential Equations. Springer, January 1992.

M A Kramer, B A Lopour, H E Kirsch, and A J Szeri. Bifurcation control of a seizing human cortex. Physical Review E, 73(4):041928, 2006.

J-S Li, J Ruths, T-Y Yu, H Arthanari, and G Wagner. Optimal pulse design in quantum control: A unified computational method. Proceedings of the National Academy of Sciences, 108(5):1879-1884, 2011.

J-S Li, I Dasanayake, and J Ruths. Control and synchronization of neuron ensembles. IEEE Transactions on Automatic Control, 58(8):1919-1930, 2013.

S-F Liang, F-Z Shaw, C-P Young, D-W Chang, and Y-C Liao. A closed-loop brain computer interface for realtime seizure detection and control. In Engineering in Medicine and Biology Society (EMBC), 2010 Annual International Conference of the IEEE, pages 4950-4953. IEEE, 2010.

S-F Liang, F-Z Shaw, D-W Chang, C-P Young, Y L Wang, and S Y Wu. Live demonstration: A portable closed-loop seizure controller in freely moving rats. In Biomedical Circuits and Systems Conference (BioCAS), 2012 IEEE, pages 88-88. IEEE, 2012.

P Limousin, P Krack, P Pollak, A Benazzouz, C Ardouin, D Hoffmann, and A-L Benabid. Electrical stimulation of the subthalamic nucleus in advanced parkinson's disease. New England Journal of Medicine, 339(16): 1105-1111, 1998.

F Marten, S Rodrigues, P Suffczynski, and M P Richardson. Derivation and analysis of an ordinary differential equation mean-field model for studying clinically recorded epilepsy dynamics. Physical Review E, 2009.

J Moehlis, E Shea-Brown, and H Rabitz. Optimal inputs for phase models of spiking neurons. Journal of Computational and Nonlinear Dynamics, 1:358, 2006.

D. Pinault and T.J. O'Brien. Cellular and network mechanisms of genetically-determined absence seizures. Thalamus 83 Related Systems, 3(3):181, 2005.

J Ruths and J-S Li. Optimal Control of Inhomogeneous Ensembles. IEEE Transactions on Automatic Control, 57(8):2021-2032, 2012.
S Saillet, S Gharbi, G Charvet, C Deransart, R Guillemaud, A Depaulis, and O David. Neural adaptation to responsive stimulation: A comparison of auditory and deep brain stimulation in a rat model of absence epilepsy. Brain Stimulation, 2012.

S J Schiff. Towards model-based control of parkinson's disease. Philosophical Transactions of the Royal Society A: Mathematical, Physical and Engineering Sciences, 368(1918):2269-2308, 2010.

P. Suffczynski, S. Kalitzin, and FH Lopes Da Silva. Dynamics of non-convulsive epileptic phenomena modeled by a bistable neuronal network. Neuroscience, 126(2): 467-484, 2004.

P A Tass. A model of desynchronizing deep brain stimulation with a demand-controlled coordinated reset of neural subpopulations. Biological cybernetics, 89(2):8188, 2003.

P N Taylor, G Baier, S S Cash, J Dauwels, J-J Slotine, and Y W. A model of stimulus induced epileptic spike-wave discharges. IEEE Symposium Series on Computational Intelligence, 2013a.

P N Taylor, Y Wang, J Dauwels, and G Baier. Abatement of epileptic spike-wave discharges through single pulse stimulation. BMC Neuroscience, 2013b.

P.N. Taylor and G. Baier. A spatially extended model for macroscopic spike-wave discharges. Journal of Computational Neuroscience, 31(3):679-684, 2011.

P.N. Taylor, M. Goodfellow, Y. Wang, and G. Baier. Towards a large-scale model of patient-specific epileptic spike-wave discharges. Biological Cybernetics, 2013c.

Y. Wang, M. Goodfellow, P.N. Taylor, and G. Baier. Phase space approach for modeling of epileptic dynamics. Physical Review E, 85(6):061918, 2012. 